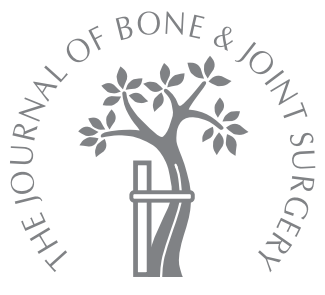

TRAUMA

\title{
A randomised pilot trial of "locking plate" fixation versus intramedullary nailing for extra-articular fractures of the distal tibia
}

\author{
C. Mauffrey, \\ K. McGuinness, \\ N. Parsons, \\ J. Achten, \\ M. L. Costa \\ From Warwick \\ Orthopaedics, \\ University of \\ Warwick, Warwick, \\ United Kingdom
}

C. Mauffrey, MD, FRCS, Assistant Professor in Orthopedic Traumatology Denver Health Medical Centre Orthopedic Department, 777 Bannock Street, Denver, Colorado 80204, USA.

K. McGuinness, BSc, Trial Coordinator

N. Parsons, PhD, Statistician U. J. Achten, $\mathrm{PhD}$, Senior Research Fellow

M. L. Costa, PhD, FRCS, Professor of Trauma and Orthopaedics

Warwick Orthopaedics, University of Warwick, Clinical Science Building, Clifford Bridge Road, Coventry CV2 2DX, UK.

Correspondence should be sent to $\mathrm{Mr}$ C. Mauffrey; e-mail: cyril.mauffrey@dhha.org

(C)2012 British Editorial Society of Bone and Joint Surgery doi:10.1302/0301-620X.94B5 $28498 \$ 2.00$

$J$ Bone Joint Surg Br 2012;94-B:704-8.

Received 15 October 2011;

Accepted after revision 12

January 2012

\begin{abstract}
The ideal form of fixation for displaced, extra-articular fractures of the distal tibia remains controversial. In the UK, open reduction and internal fixation with locking-plates and intramedullary nailing are the two most common forms of treatment. Both techniques provide reliable fixation but both are associated with specific complications. There is little information regarding the functional recovery following either procedure.

We performed a randomised pilot trial to determine the functional outcome of 24 adult patients treated with either a locking-plate $(n=12)$ or an intramedullary nailing $(n=12)$. At six months, there was an adjusted difference of 13 points in the Disability Rating Index in favour of the intramedullary nail. However, this was not statistically significant in this pilot trial ( $p=0.498$ ). A total of seven patients required further surgery in the locking-plate group and one in the intramedullary nail group.

This study suggests that there may be clinically relevant, functional differences in patients treated with nail versus locking-plate fixation for fractures of the distal tibia and differences in related complications. Further trials are required to confirm the findings of this pilot investigation.
\end{abstract}

The mechanism of injury and the prognosis of displaced, extra-articular fractures of the distal tibia is different to that for pilon fractures. Their proximity to the ankle, however, makes the surgical treatment more complicated than the treatment of diaphyseal fractures of the tibia. ${ }^{1}$ Locked intramedullary nailing is considered the treatment of choice for diaphyseal fractures but there are concerns about the stability of the fixation, breakage of the nail and locking screws, risk of propagation of the fracture into the ankle joint, and unsatisfactory alignment in fractures involving the metaphysis of the tibia. ${ }^{2,3}$ In the United Kingdom, closed and Gustilo ${ }^{4}$ extra-articular fractures of the distal tibia are most commonly managed with intramedullary (IM) nailing or with a plate and fixed-angle screws (locking-plate). ${ }^{5}$ Previous investigations have suggested differences in the rate of complications and time to healing after these different types of fixation, ${ }^{5-7}$ but have not compared outcome. We therefore performed a randomised pilot trial to compare these forms of fixation in the treatment of fractures of the distal tibia in adults. We used a patient-reported functional outcome tool (Disability Rating Index ${ }^{8}$ : DRI) as the primary outcome measure.

\section{Patients and Methods}

The trial was approved by the Local Research Ethics Committee and appropriately registered on the ISRCTN database. Adult patients aged 18 years or over with a closed or Gustilo I extra-articular fracture of the distal tibia were recruited from a large teaching hospital. The distal tibia was defined as the area within two Müller squares ${ }^{9}$ of the ankle joint, in which the proximal and the distal segments of long bones are defined by a square whose sides have the same length as the widest part of the epiphysis.

The fracture was diagnosed on orthogonal radiographs. CT was only used if intra-articular extension was suspected. Patients were excluded if they had other injuries which could affect the primary outcome measure, such as polytrauma or vascular or neurological injuries, if the fracture was too distal to achieve fixation of four cortices with distal interlocking screws, if the patient had a history of peripheral vascular disease or had a contraindication to anaesthesia. After consent, patients were randomly allocated to the two treatment groups in a 1:1 ratio. Treatment allocation was determined using a computer-generated pseudo-random number sequence and administered by an independent trial co-ordinator (KMG) who was contacted by telephone.

In this pragmatic trial, the surgery was supervised by one of eight Consultant Orthopaedic Surgeons (including MLC). In general, 
the locking-plates were applied using indirect reduction techniques and bridge-plating and the nails were secured using a minimum of two distal non-locking screws. However, the surgeons were given the freedom to adapt these techniques as required by the configuration of the fracture and in accordance with their preferred technique and surgical approach. Patients in both groups were encouraged to start range of movement exercises at the knee and the ankle as soon as possible. Progression to full-weight-bearing on the affected leg was permitted as comfort allowed but again the details of the post-operative rehabilitation regime were determined by the treating surgeon in conjunction with the physiotherapist.

The primary outcome measure was the DRI,${ }^{8}$ which is a validated 12-item, patient reported, functional outcome questionnaire that is transformed to a 100 point scale, where 0 represents normal function and 100 complete disability. At an individual patient level, a difference of eight points represents the ability to climb stairs or run with 'some difficulty' versus 'great difficulty'. At a population level, eight points represents the difference between a 'healthy patient' and a 'patient with a minor disability'. ${ }^{8}$

Secondary outcome measures were the Olerud and Molander Ankle Score (OMAS) ${ }^{10}$ and the EuroQol EQ-5D generalised health outcome questionnaire. ${ }^{11}$ The OMAS is another patient reported score but is more specifically related to injuries around the ankle and includes assessment of: pain, stiffness, swelling, stair climbing, running, jumping, squatting, supports and work and activities of daily living. It is transformed to a 100 point scale, where 100 represents normal function and 0 the worst possible function. The EuroQol EQ-5D is a validated measure of health-related quality of life, consisting of a five-dimension health status classification system and a separate visual analogue scale measuring the pain level on a scale from 0 to 10 , with 10 being the worst pain they ever had. In addition, union of the fracture was assessed using the three criteria described by Sarmiento et $\mathrm{al}^{12}: 1$ ) the ability of the patient to bear weight without pain, 2) the absence of clinically detectable movement across the fracture, and 3) visible bridging callus across the fracture on the radiograph. Normal time to union was considered to be 24 weeks. Mal- or nonunion was defined as a varus or valgus deformity $>5^{\circ}$, an anterior/posterior angulation $>10^{\circ}$, a rotational deformity $>10^{\circ}$, and shortening $>10 \mathrm{~mm} .{ }^{13}$ All subsequent operations related to the fracture or complications of the injury were recorded.

The outcome data were collected by an experienced research physiotherapist (KMG) blinded to treatment allocation, at six weeks, three, six and 12 months post-operatively. A total of 12 patients were randomised into each group between March 2008 and August 2009. Patient flow through the study can be seen in Figure 1 .

Statistical analysis. The main analysis investigated differences in the primary outcome measure (DRI) between the two treatment groups on an intention-to-treat basis at six months post-operatively. Six months was chosen as the point at which a patient making an uncomplicated recovery would be expected to return to their normal daily activities. However, all patients were followed up for a minimum of 12 months to record any late complications, specifically nonunion. As this was a pilot study, the analysis was exploratory in nature, with the primary aim to assess the size and direction of observed differences between the treatment groups. No formal sample size calculation was performed but a sample of 24 patients in total was considered to be sufficient to assess variability between patients and crudely estimate the nature of any treatment effect; this was a small study, so the size of the treatment effect was unlikely to be estimated with much precision. However, formal significance testing was undertaken, assuming an approximate normal distribution for the DRI scores at six months and 12 months post-operation, using Student's $t$-test and linear regression analysis to adjust for imbalance in the baseline characteristics between treatment groups. Diagnostic quantile-quantile plots of the residuals were used to assess the normality assumptions. Statistical significance was set at the 5\% level. Analyses and graphical summaries were produced using the statistical package R (R Foundation for Statistical Computing, Vienna, Austria).

\section{Results}

One patient allocated to the IM nail group was subsequently treated non-operatively due to the peri-operative exacerbation of a pre-existing comorbidity. The patient had an exacerbation of their emphysema and it was decided to treat the fracture non-surgically. The two groups of patients were reasonably well-balanced in terms of gender, body mass index (BMI), OMAS and EQ-5D (Table I). However, the mean age of the IM nail group was older than the plate group and consequently it was necessary to adjust for this factor in the analysis.

In the IM nail group, all patients had two distal locking screws in the coronal plane. In addition, two patients had a third locking screw inserted in the sagittal plane. 'Blocking' (Poller) screws were used in four cases. ${ }^{14}$ The fibula was fixed with a plate and screws in two cases.

In the plate group, all patients had between four and six distal screws inserted through the plate. Supplementary screws (non-locking) were used in four cases either through the plate or separately. The fibula was not fixed in any of the patients in this group.

Post-operative functional outcome measures. Temporal trends for DRI, OMAS and EQ-5D (Figs 2 to 4) show differences that persisted from six to 12 months post-operatively for all these measures. The mean DRI scores in the IM nail group were 32.1 (95\% confidence interval (CI); 15.4 to 48.9 ) and 23.4 (95\% CI 4.2 to 42.6$)$ at six and 12 months and for the plate group were $39.2(95 \%$ CI 24.2 to 54.2$)$ and 27.3 (95\% CI 9.3 to 45.2 ) at six and 12 months. There was no statistically significant difference in these scores between the groups at either time (six months, $\mathrm{p}=0.498$; 


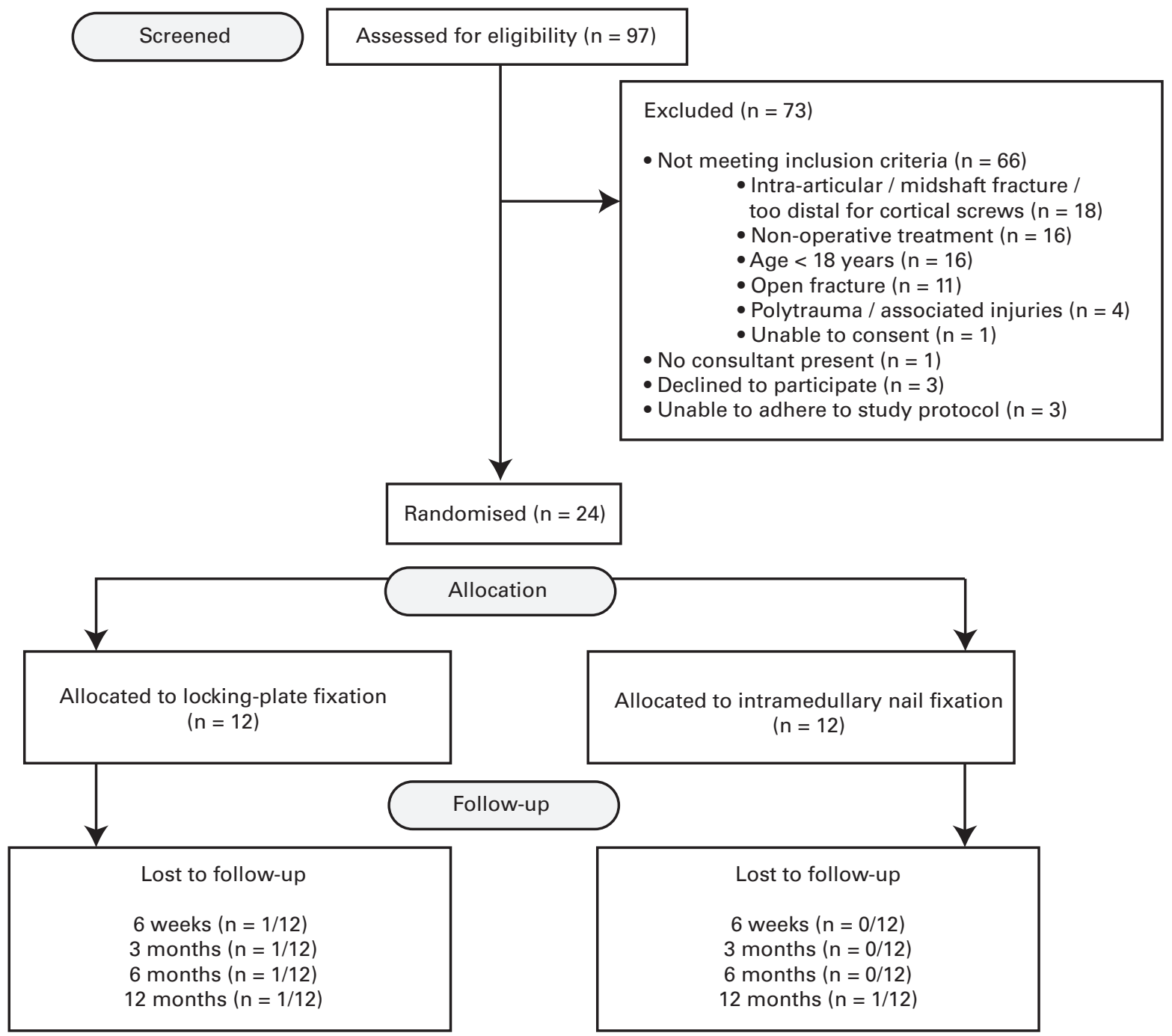

Fig. 1

Consort flow diagram.

Table I. Patient baseline characteristics. Data shown for all characteristics, other than gender, are medians and interquartile ranges (IQR)

\begin{tabular}{lll}
\hline & \multicolumn{2}{l}{ Treatment group } \\
\cline { 2 - 3 } Characteristic $^{*}$ & Nail ( $\mathbf{n = 1 2})$ & Plate $(\mathbf{n}=12)$ \\
\hline Male:female & $7: 5$ & $9: 3$ \\
Age (yrs) & $50(39$ to 60$)$ & $33(24$ to 43$)$ \\
Body mass index $\left(\mathrm{kg} / \mathrm{m}^{2}\right)$ & $24.3(21.5$ to 26.7$)$ & $23.4(22.7$ to 25.6$)$ \\
OMAS (0 to 100$)$ & $100(100$ to 100$)$ & $100(98$ to 100$)$ \\
EQ-5D score & $1.00(0.84$ to 1.00$)$ & $1.00(0.96$ to 1.00$)$ \\
EQ-5D VAS $(0$ to 100$)$ & $80(75$ to 90$)$ & $93(78$ to 100$)$ \\
DRI (0 to 100) & $0.2(0.0$ to 20.2$)$ & $0.0(0.0$ to 2.2$)$ \\
\hline
\end{tabular}

* OMAS, Olerud and Molander Ankle Score; DRI, Disability Rating Index; EQ-5D, EuroQol EQ-5D

12 months, $\mathrm{p}=0.743 ; t$-test). The difference in the mean DRI scores at six months was approximately 7 points. However, a linear regression analysis that accounted for the difference in patient age between groups indicated that the true (adjusted) difference in mean DRI scores between groups at six months was approximately 13 points (regression coefficient $(\beta)=13.1$ with standard error (SE) 9.7). Age at baseline was significantly associated with DRI score at six months $(\beta=0.62$ ( $\mathrm{SE}=0.27)$, and $\mathrm{z}$-test from regression analysis with $\mathrm{p}=0.03$ ); older patients had higher DRI scores at six months. A quantile-quantile plot, and the symmetry of the six month boxplots shown in Figure 2, indicated that the assumption of approximate normality for DRI at six months was acceptable. Thus, the observed 7 point difference in mean DRI was an underestimate of the true treatment effect, as the older patients in the IM nail group raised the mean DRI score relative to the younger patients in the plate group. The estimated 13-point difference in the mean DRI at six months is greater than the minimum clinically important difference for this outcome measurement tool; in other words, a difference of this magnitude is clinically relevant to this group of patients. 


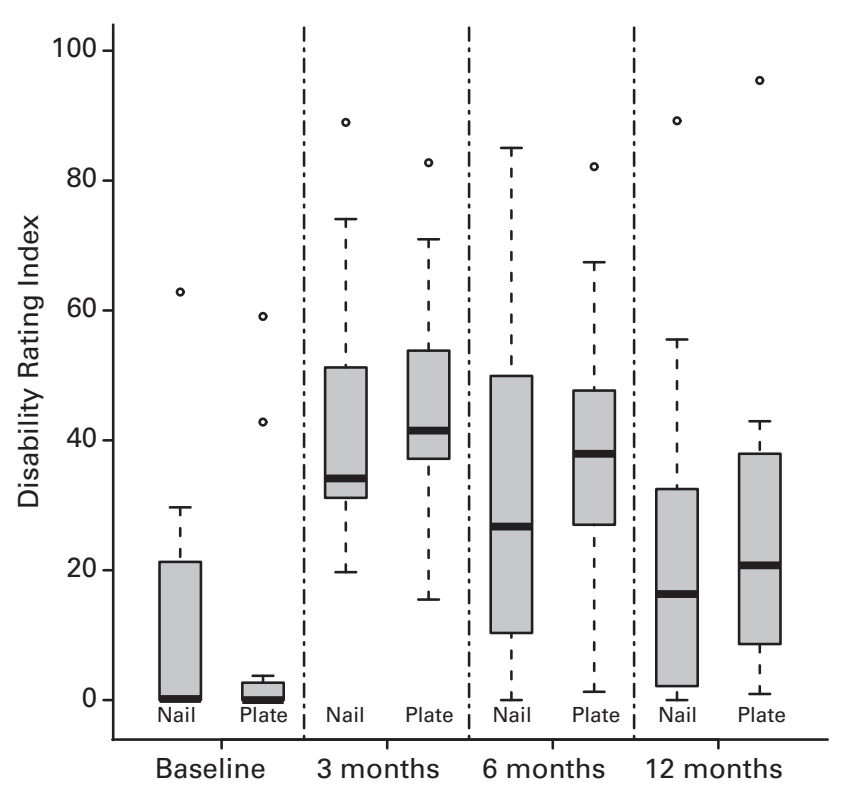

Fig. 2

Boxplots showing the Disability Rating Index (DRI) for both groups at baseline, three months, six months and 12 months. The boxes represent the median and interquartile range, and the whiskers the range of data excluding outliers $\left(^{\circ}\right)$

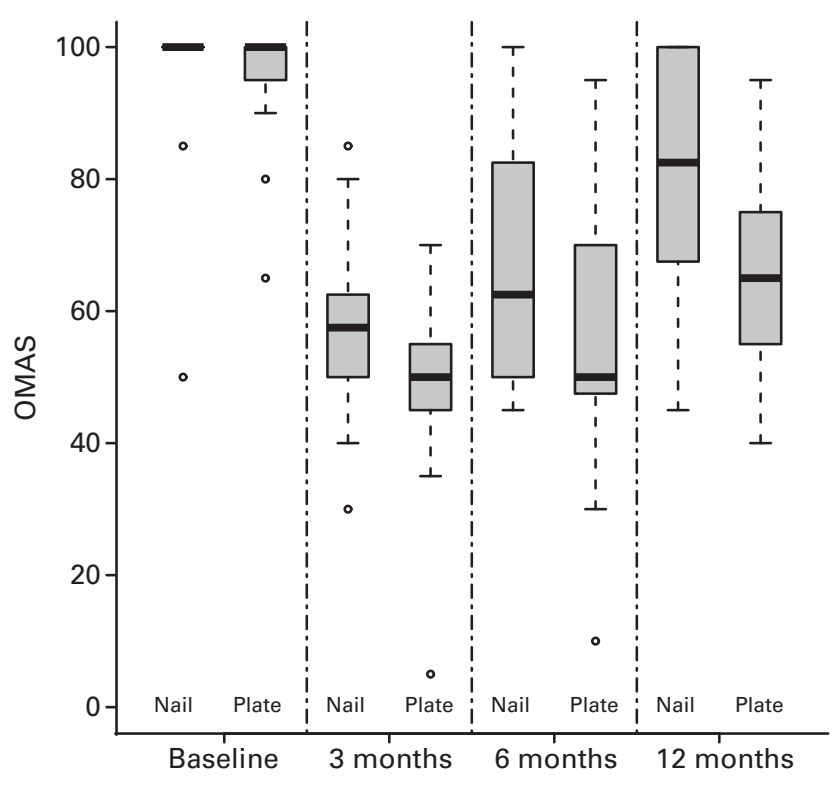

Fig. 3

Boxplots showing the Olerud and Molander Ankle Score (OMAS) for both groups at baseline, three months, six months and 12 months. The boxes represent the median and interquartile range, and the whiskers the range of data excluding outliers $\left(^{\circ}\right)$.

The complications in the two groups are detailed in Table II. There was only one deep infection, which occurred in the plate group and was successfully managed with debridement, antibiotics and subsequent removal of the

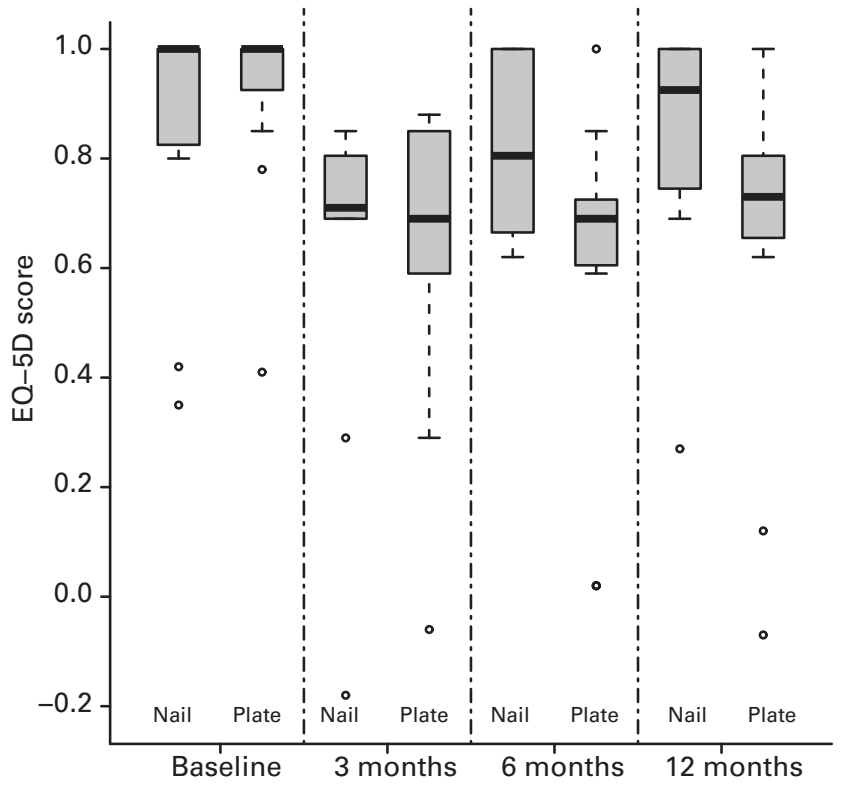

Fig. 4

Boxplots showing the EuroOol EQ-5D scores for both groups at baseline, three months, six months and 12 months. The boxes represent the median and interquartile range, and the whiskers the range of data excluding outliers $\left(^{\circ}\right)$.

Table II. Complications

\begin{tabular}{lll}
\hline & \multicolumn{2}{l}{ Treatment group } \\
\cline { 2 - 3 } Complication & Nail $(\mathbf{n}=\mathbf{1 2})$ & Plate $(\mathbf{n}=\mathbf{1 2})$ \\
\hline Delayed union ( $>24$ weeks) & 1 & 3 \\
Removal of metalwork & 1 & 4 \\
$\begin{array}{l}\text { Delayed wound healing/superficial } \\
\text { infection }\end{array}$ & 3 & 0 \\
$\begin{array}{l}\text { Deep infection requiring surgical } \\
\text { debridement }\end{array}$ & 0 & 1 \\
$\begin{array}{l}\text { Buckled plate } \\
\text { Compartment syndrome }\end{array}$ & 0 & 1 \\
\hline
\end{tabular}

plate when the fracture had united. There were three superficial wound infections, all of which were related to the locking screws in the IM nail group. These resolved with oral antibiotics. One plate buckled when the patient jumped from a bed in the immediate post-operative period, within 48 hours. The fixed-angle fixation was intact; he underwent a manipulation under anaesthetic, which restored the alignment of the tibia. There were three delayed unions in the plate group: one fracture eventually united unremarkably by eight months, one was revised to an IM nail and united by seven months and one nonunion is awaiting further surgery. One delayed union in the IM nail group eventually united without intervention at seven months. Three plates and one IM nail were subsequently removed because of local symptoms. There was one malunion ( $10^{\circ}$ of valgus) in the nail group. There were two cases 
of compartment syndrome which required fasciotomies one in each group.

\section{Discussion}

In this pilot investigation, we did not find any evidence of a statistically significant difference in functional outcome scores between patients treated with a locking-plate and those treated with an IM nail for an extra-articular fracture of the distal tibia. However, at six months post-operatively, there was an estimated 13-point (SD 24) difference in the primary outcome measure in favour of the IM nail group, after adjusting for the age differences between the groups. This difference persisted after this time-point but began to diminish by the 12-month assessment, suggesting that there might be clinically meaningful differences in functional outcome between these two forms of treatment. Clearly, caution must be observed in interpreting differences from pilot studies with small treatment group sizes, such as those used here.

In terms of complications, there were more cases of delayed union in the locking-plate group, two of which required further surgery. This may be due to biological or mechanical factors, both of which may be related to surgical technique as much as to the implants, but this study was not designed to investigate this area specifically. The three cases of superficial wound complications in the nailing group were all related to the site of insertion of the locking screws, but the only deep infection occurred in the plate group. Metalwork was not removed routinely, but four patients in the plate group and one in the IM nail group had symptomatic metalwork removed.

There are two previous randomised trials in this area. Im and $\mathrm{Tae}^{6}$ performed a trial of IM nailing versus traditional (non-locking) plate fixation for the management of extraarticular fractures of the distal tibia. They recruited 64 patients and followed them for two years. The difference in the primary outcome measure (OMAS) between the two groups was not statistically significant that time. The IM nailing group had a better range of movement and fewer wound problems, with one superficial infection in the nailing group and six superficial and one deep infection in the ORIF group ( $p=0.03$ ). However, the plate group had better overall alignment $(\mathrm{p}=0.01)$. Guo et $\mathrm{al}^{7}$ performed a randomised controlled trial of 85 patients treated with IM nail versus locking-plate fixation for the management of extra-articular fractures of the distal tibia. This study showed that the two groups were similar in the amount of residual pain, alignment and function as measured by the American Academy Foot and Ankle score (AAFAS) ${ }^{15}$ at one year. However, the AAFAS has limited validation data and is unlikely to reflect the treatment priorities relevant to patients with an extra-articular fracture of the tibia. The operating time and radiation administered intra-opera- tively were significantly less in the IM nailing group. The IM nail group also had fewer wound problems (three patients $(6.8 \%)$ in the IM nail group and six $(14.6 \%)$ in the locking-plate group). In that study, metalwork was regularly removed, but less commonly in the IM nail group (37 patients $(84.1 \%)$ in the nail group and 38 patients $(92.7 \%)$ in the plate group expressed a wish to have the implant removed).

The strengths of our study include the use of a validated, functional primary outcome measure and the pragmatic study design, involving several surgeons and operative techniques. The key weakness was the small sample size. This pilot trial was not statistically designed to provide a definitive answer with regard to the optimal fixation for patients with a fracture of the distal tibia. However, it does provide a compelling rationale for a further larger trial.

This study was supported by the AO Foundation Project Number S-07-4C.

No benefits in any form have been received or will be received from a commercial party related directly or indirectly to the subject of this article.

\section{References}

1. Robinson CM, McLauchlan GJ, McLean IP, Court-Brown CM. Distal metaphyseal fractures of the tibia with minimal involvement of the ankle: classification and treatment by locked intramedullary nailing. J Bone Joint Surg [Br] 1995;77-B:781787

2. Boenisch UW, de Boer PG, Journeaux SF. Unreamed intramedullary tibial nailing: fatigue of locking bolts. Injury 1996;27:265-270.

3. Mosheiff R, Safran $\mathbf{0}$, Segal D, Liebergall M. The undreamed tibial nail in the treatment of distal metaphyseal fractures. Injury 1999;30:83-90.

4. Gustilo RB, Anderson JT. Prevention of infection in the treatment of one thousand and twenty-five open fractures of long bones: retrospective and prospective analyses. J Bone Joint Surg [Am] 1976;58-A:453-458.

5. Zelle BA, Bhandari M, Espiritu M, Koval KJ, Zlowodzki M; Evidence-Based Orthopaedic Trauma Working Group. Treatment of distal tibia fractures without articular involvment: a systematic review of 1125 fractures. J Orthop Trauma 2006;20:76-79

6. Im GI, Tae SK. Distal metaphyseal fractures of tibia: a prospective randomized trial of closed reduction and intramedullary nail versus open reduction and plate and screws fixation. J Trauma 2005;59:1219-1223.

7. Guo JJ, Tang N, Yang HL, Tang TS. A prospective, randomised trial comparing closed intramedullary nailing with percutaneous plating in the treatment of distal metaphyseal fractures of the tibia. J Bone Joint Surg [Br] 2010;92-B:984-988.

8. Salén BA, Spangfort EV, Nygren AL, Nordemar R. The Disability Rating Index: an instrument for the assessment of disability in clinical settings. J Clin Epidemiol 1994:47:1423-1435

9. Müller ME, Nazarian S, Koch P, Schatzker J. The comprehensive classification of fractures of long bones. First ed. Berlin Heidelberg New York: Springer-Verlag, 1990.

10. Olerud C, Molander H. A scoring scale for symptom evaluation after ankle fracture. Arch Orthop Trauma Surg 1984;103:190-194.

11. No authors listed. Euro0ol: a new facility for the measurement of health-related quality of life: the Euro0ol Group. Health Policy 1990;16:199-208.

12. Sarmiento A, Sobol PA, Sew Hoy AL, et al. Prefabricated functional braces for the treatment of fractures of the tibial diaphysis. J Bone Joint Surg [Am] 1984;66A:1328-1339.

13. Milner SA. A more accurate method of measurement of angulation after fractures of the tibia. J Bone Joint Surg [Br] 1997;79-B:972-974.

14. Stedfeldt HW, Mittlmeier T, Landgraf P, Ewert A. The logic and clinical applications of blocking screws. J Bone Joint Surg [Am] 2004;86-A(Suppl 2):17-25.

15. American Academy of Orthopaedic Surgeons. Lower extremity outcomes instruments and information. http://www.aaos.org/research/outcomes/outcomes_lower.asp (date last accessed 20 February 2011). 